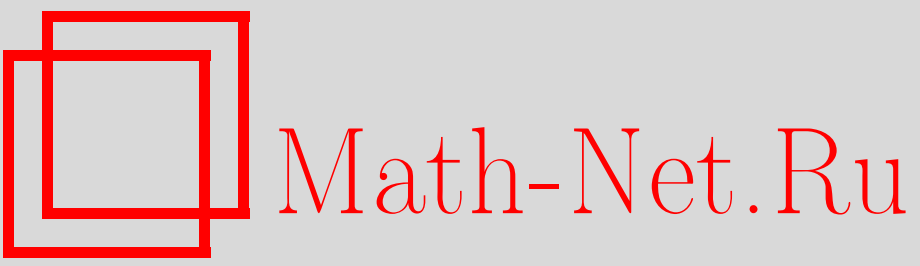

Г. Ганболд, Запаздывающий функционал действия для дираковского полярона, TMФ, 2000, том 122, номер 2, 231-238

DOI: https://doi.org/10.4213/tmf565

Использование Общероссийского математического портала Math-Net.Ru подразумевает, что вы прочитали и согласны с пользовательским соглашением

http://www.mathnet.ru/rus/agreement

Параметры загрузки:

IP: 3.95 .254 .165

26 апреля 2023 г., 18:06:31 
ТЕОРЕТИЧЕСКАЯ

И МАТЕМАТИЧЕСКАЯ

ФИЗИКА

Том 122, № 2

февраль, 2000

(C) 2000 г.

Г. Ганболд*

\section{ЗАПАЗДЫВАЮЩИЙ ФУНКЦИОНАЛ ДЕЙСТВИЯ ДЛЯ ДИРАКОВСКОГО ПОЛЯРОНА}

Характеристики основного состояния одномерного (дираковского) полярона рассматриваются в качестве примера системы с запаздывающим функционалом взаимодействия. В подходе континуального интеграла получены оценки энергии основного состояния и эффективной массы полярона в рамках и вне рамок наиболее общего гауссова приближения. Показано, что ведуший гауссов вклад в собственную энергию немного улучшает фейнмановскую оценку, при этом он принадлежит самой нижней из возможных верхних границ. Негауссовы поправки следующего за ведущим порядка не нарушают значительно полученных результатов. Для сравнения была вычислена нижняя граница энергии основного состояния полярона в рамках метода Либа-Ямадзаки.

\section{1. ВВЕДЕНИЕ}

Электрон, движушийся в ионном кристалле, и сопровождаюшее это движение искажение поляризованной решетки называются поляроном [1-3]. В последнее время существенное внимание исследователей привлекали свойства поляронов, удерживаемых в одномерии $(d=1)$. Растущий интерес к низкоразмерным поляронам может быть обусловлен рядом причин, среди которых сушественное место занимает то обстоятельство, что современная полупроводниковая технология [4-7] сделала возможным удержание электронов в квазинизкоразмерных структурах. Другая причина связана с неизбежным теоретическим улучшением стандартной $(d=3)$ модели полярона путем понижения пространственной размерности [8-11].

Результируюшее взаимодействие с поляризованной решеткой изменяет как энергию, так и массу электрона. В силу этого основные характеристики полярона как квазичастищы, такие как энергия основного состояния (ЭОС) и эффективная масса (ЭМ), зависят от безразмерной константы связи $\alpha$.

В настоящей работе мы сосредоточимся на получении точных и приближенных результатов для ЭОС и ЭМ одномерного аналога большого полярона Фрёлиха при произвольном значении константы связи $0 \leqslant \alpha \leqslant \infty$.

*Institut für Theoretische Physik, Universität Erlangen-Nürnberg, Erlangen, Germany 


\section{2. ПОЛЯРОННЫЙ ИНТЕГРАЛ ПО ТРАЕКТОРИЯМ В ОДНОМЕРНОМ СЛУЧАЕ}

Дираковский $(d=1)$ полярон описывается короткодействующим потенциалом типа $\delta$-функции, тогда как полярон Фрёлиха $(d=3)$ участвует во взаимодействии кулоновского типа - дальнодействии. Результируюшее взаимодействие электрона, удерживаемого в одномерной системе, с фононами продольных оптических (LO-моды) колебаний решетки моделируется с помошью следуюшего гамильтониана [12]:

$$
H=\frac{p^{2}}{2}+\sum_{k} a_{k}^{\dagger} a_{k}+\sqrt{\frac{2 \alpha}{L}} \sum_{k} g_{k}\left(a_{k}^{\dagger} e^{-i k r}-a_{k} e^{i k r}\right),
$$

где $p$ и $r$ - импульс и положение электрона, $L$ - длина кристалла решетки, а $k, a_{k}$ и $a_{k}^{\dagger}-$ соответственно волновой вектор и операторы уничтожения и рождения фононов. Здесь мы использовали единишы, в которых $m_{b}=\omega_{\mathrm{LO}}=\hbar=c=1$, где $m_{b}$ - масса затравочного электрона, $\omega_{\mathrm{LO}}$ обозначает частоту LO-моды решеточных колебаний. Безразмерная константа взаимодействия $\alpha$ выбрана так, что $\lim _{\alpha \rightarrow 0} E(\alpha) / \alpha=1$.

Линейный характер электрон-фононного взаимодействия в гамильтониане (1) позволяет проинтегрировать по фононам в рамках знаменитого фейнмановского формализма интегралов по траекториям (ИТ) [13]. Поляронная ЭОС определяется тогда как

$$
\begin{gathered}
E(\alpha):=-\lim _{\beta \rightarrow \infty} \frac{1}{\beta} \ln Z_{\beta}(\alpha, x), \quad Z_{\beta}(\alpha, x):=\int \mathcal{D} r \delta(r(\beta)-x) e^{\alpha \Phi_{\beta}(r),} \\
\Phi_{\beta}(r):=\frac{1}{\sqrt{8}} \iint_{0}^{\beta} d t d s e^{-|t-s|} \delta(|r(t)-r(s)|),
\end{gathered}
$$

где $\mathcal{D} r$ - нормированная функциональная мера винеровского типа, определенная на траекториях, начинаюшихся в начале координат, $r(0)=0$, а $\delta(r)-\delta$-функция.

ЭМ полярона можно определить, используя разложение вешественной части собственной энергии по малому фиксированному импульсу $p$ :

$$
m^{*}(\alpha):=-\left\{\left.\frac{\partial^{2}}{\partial p^{2}} \lim _{\beta \rightarrow \infty} \frac{1}{\beta} \ln \left(\int d x e^{-i p x} Z_{\beta}(\alpha, x)\right)\right|_{|p|=0}\right\}^{-1} .
$$

Определения (2)-(4) подчиняются условиям нормировки $E(0)=0$ и $m^{*}(0)=1$.

ИТ $Z_{\beta}$ является центральным объектом в нашем рассмотрении, обычно его удается вычислить явно лишь в предельных случаях слабого и сильного взаимодействий. Точные асимптотические выражения

$$
\begin{aligned}
E(\alpha) & = \begin{cases}-\alpha-\alpha^{2}\left(\frac{3}{\sqrt{8}}-1\right)+O\left(\alpha^{3}\right), & \alpha \rightarrow 0, \\
-\frac{\alpha^{2}}{3}+O(1), & \alpha \rightarrow \infty,\end{cases} \\
m^{*}(\alpha) & = \begin{cases}1+\frac{\alpha}{2}+\frac{\alpha^{2}(5 \sqrt{2}-4)}{16}+O\left(\alpha^{3}\right), & \alpha \rightarrow 0, \\
\frac{32 \alpha^{4}}{15}+O\left(\alpha^{2}\right), & \alpha \rightarrow \infty,\end{cases}
\end{aligned}
$$

можно найти в работах $[11,14,12,10]$. 


\section{3. ГАУССОВЫ ВКЛАДЫ ВЕДУШЕГО ПОРЯДКА}

Как было отмечено выше, точные результаты обычно удается получить только в предельных случаях $\alpha \rightarrow 0$ и $\alpha \rightarrow \infty$. В случае других значений $(\alpha \sim 1)$ для оценок ЭОС и ЭМ полярона были развиты различные приближенные методы [15-17].

Верхние границы в гауссовом приближении. Функционал поляронного взаимодействия $\Phi_{\beta}(r)(3)$ является вещественным. Экспонента в выражении (2) остается выпуклой функцией для любого вещественного пробного функционала $\Phi_{\beta}^{\mathrm{tr}}(r)$, введенного вместо $\Phi_{\beta}(r)$. Тогда соответствующий пробный функционал $Z_{\beta}^{\operatorname{tr}}(\alpha, 0)$ является интегрируемым, если выбрать $\Phi_{\beta}^{\mathrm{tr}}(r)$ квадратичным. Эти обстоятельства дают возможность применить к $Z_{\beta}^{\mathrm{tr}}(\alpha, 0)$ равенство Иенсена и получить верхнюю границу ЭОС. Детали и результаты такого приближения зависят, конечно, от специального выбора пробного функционала. Ниже мы кратко рассмотрим два подхода, основанные на принципе вариационной оптимизации, с целью получения приемлемой верхней границы ЭОС одномерного полярона. После этого мы применим к данной задаче невариационный метод $(\mathrm{GER})^{1)}$, дающий разумное значение верхней границы ЭОС.

1. В простейшем приближении следует рассмотреть функционал $Z_{\beta}^{\operatorname{tr}}(\alpha, 0)$, подходящий для гармонического осциллятора с зависяшей от $\alpha$ массой $\mu(\alpha)$. "Осцилляторные" приближения для ЭОС и ЭМ имеют вид

$$
\begin{aligned}
& E_{\mathrm{OS}}(\alpha)=\inf \left\{\frac{\mu}{4}-\frac{\alpha \sqrt{\mu}}{\sqrt{\pi}} \int_{0}^{\infty} d t \frac{e^{-t}}{\left(1-e^{-\mu t}\right)^{\frac{1}{2}}}\right\} \\
& m_{\mathrm{oS}}^{*}(\alpha)=1+\frac{\alpha \mu^{\frac{3}{2}}}{\sqrt{\pi}} \int_{0}^{\infty} d t \frac{t^{2} e^{-t}}{\left(1-e^{-\mu t}\right)^{\frac{3}{2}}}
\end{aligned}
$$

где параметр $\mu$ доставляет минимум выражению в фигурных скобках. Результаты оценок для $E_{\mathrm{OS}}(\alpha) \geqslant E(\alpha)$ приведены в табл. 1 и 2 . Одним из недостатков данной модели является искусственная сингулярность, появляюшаяся в высших производных $E_{\mathrm{OS}}(\alpha)$ в точке $\alpha \cong 1.93$.

2. При получении своей знаменитой оценки (в случае $d=3$ ) Фейнман использовал двухпараметрическое квадратичное пробное действие, моделирующее запаздывающее взаимодействие осцилляторного типа между поляроном и массивной частицей. Применяя данный подход при $d=1$ и оптимизируя пробную собственную энергию по параметрам $v>w>1$, получаем следующие выражения для вариационной верхней границы ЭОС и ЭМ фейнмановского типа [9]:

$$
\begin{aligned}
& E_{\mathrm{F}}(\alpha)=\inf \left\{\frac{(v-w)^{2}}{4 v}-\frac{\alpha v^{\frac{3}{2}}}{\sqrt{\pi}} \int_{0}^{\infty} d t \frac{e^{-t}}{\left[w^{2} v t+\left(v^{2}-w^{2}\right)\left(1-e^{-v t}\right)\right]^{\frac{1}{2}}}\right\}, \\
& m_{\mathrm{F}}^{*}(\alpha)=1+\frac{\alpha v^{\frac{5}{2}}}{\sqrt{\pi}} \int_{0}^{\infty} d t \frac{t^{2} e^{-t}}{\left[w^{2} v t+\left(v^{2}-w^{2}\right)\left(1-e^{-v t}\right)\right]^{\frac{3}{2}}} .
\end{aligned}
$$

\footnotetext{
${ }^{1)}$ Gauss Equivalent Representation.
} 
Результаты, пересчитанные для промежуточных значений $\alpha$, приведены в табл. 1 и 2 для сравнения с нашими оценками. Гладкая верхняя граница $E_{\mathrm{F}}(\alpha) \geqslant E(\alpha)$ остается одной из лучших оценок при всех значениях констант связи.

ТАБЛИЦА 1

Верхняя и нижняя границы ЭОС одномерного полярона в области промежуточных значений константы связи $\alpha$

\begin{tabular}{|c|c|c|c|c|c|}
\hline$\alpha$ & $E_{\mathrm{OS}}$ & $E_{\mathrm{F}}$ & $E_{0}$ & $E_{2}$ & $E_{\mathrm{LY}}$ \\
\hline 0.5 & -0.5000 & -0.51006 & -0.51027 & -0.51589 & -0.58263 \\
1.0 & -1.0000 & -1.04444 & -1.04532 & -1.06710 & -1.44082 \\
1.5 & -1.5000 & -1.61314 & -1.61522 & -1.66119 & -2.72255 \\
2.0 & -2.0086 & -2.23696 & -2.24044 & -2.31150 & -4.48444 \\
2.5 & -2.7043 & -2.95968 & -2.96352 & -3.05052 & -6.74002 \\
4.0 & -5.7934 & -6.04779 & -6.04964 & -6.20592 & -16.4961 \\
6.0 & -12.155 & -12.4074 & -12.4083 & -12.7345 & -36.4983 \\
8.0 & -21.067 & -21.3178 & -21.3183 & -21.8871 & -64.4990 \\
\hline
\end{tabular}

ТАБЛИцА 2

ЭМ одномерного полярона в области промежуточных значений константы связи $\alpha$

\begin{tabular}{|c|c|c|c|c|c|}
\hline$\alpha$ & $m_{\mathrm{OS}}^{*}$ & $m_{\mathrm{F}}^{*}$ & $m_{0}^{*}$ & $m_{2}^{*}$ & $m_{\mathrm{LY}}^{*}$ \\
\hline 0.5 & 1.25000 & 1.32084 & 1.32246 & 1.31091 & 2.69494 \\
1.0 & 1.50000 & 1.88895 & 1.89862 & 1.83258 & 17.8088 \\
1.5 & 1.75000 & 3.11732 & 3.15094 & 2.91361 & 82.5172 \\
2.0 & 5.85933 & 6.83836 & 6.90814 & 6.13458 & 257.327 \\
2.5 & 31.6793 & 21.4723 & 21.4372 & 19.4943 & 626.221 \\
4.0 & 331.719 & 281.622 & 281.474 & 295.800 & 4097.09 \\
6.0 & 1911.78 & 1784.90 & 1784.82 & 1973.85 & 20737.0 \\
8.0 & 6302.70 & 6068.77 & 6068.73 & 6821.14 & 65537.0 \\
\hline
\end{tabular}

3. Улучшение фейнмановской оценки верхней границы можно получить путем построения более общего гауссова приближения $Z_{\beta}$ [15-17]. Ключевая идея метода GER [16] заключается в использовании представления, наиболее полно выделяюшего гауссову 
составляюшую в ИТ. Это достигается с помощью канонических преобразований, которые подчинены следующим требованиям: новая часть поляронного действия, отвечающая за взаимодействие, во-первых, обрашается в нуль при усреднении по новой мере и, во-вторых, не содержит линейных и квадратичных конфигураций путей. Эти требования определяют уравнения связи, которым подчиняется новая нормированная гауссова мера $d \sigma(r)$, введенная вместо исходной меры $\mathcal{D} r$.

Применение метода GER к соотношению (3) дает

$$
Z_{\beta}(\alpha, x)=e^{-\beta \mathcal{F}_{G}(x, \alpha, \beta)} J_{\beta}(\alpha, x),
$$

где экспоненциальный член $e^{-\beta \mathcal{F}_{G}(x, \alpha, \beta)}$ представляет собой общее гауссово приближение, а негауссовы поправки могут быть получены путем вычисления новых ИТ $J_{\beta}(\alpha, x)$ (подробнее о применении метода GER для оценки ЭОС и ЭМ полярона см. в работах $[18,16,19])$.

Опуская детали вычислений, запишем обшие гауссовы вклады в ЭОС и ЭМ в виде

$$
\begin{aligned}
& E_{0}(\alpha)=-\frac{1}{2 \pi} \int_{0}^{\infty} d k\left[\ln \left(k^{2} \widetilde{D}(k)\right)-k^{2} \widetilde{D}(k)+1\right]+\frac{\alpha}{\sqrt{2 \pi}} \int_{0}^{\infty} d t \frac{e^{-t}}{F^{\frac{1}{2}}(t)}, \\
& m_{0}^{*}(\alpha)=1+\frac{\alpha}{2 \sqrt{2 \pi}} \int_{0}^{\infty} d t \frac{t^{2} e^{-t}}{F^{\frac{3}{2}}(t)},
\end{aligned}
$$

где $\widetilde{D}(k)$ - фурье-образ функции Грина $D(t)$. Подгоночная функция $F(t):=D(0)-D(t)$, играюшая ключевую роль в новом представлении (8), должна определяться из уравнений связи

$$
F(t)=\frac{1}{\pi} \int_{0}^{\infty} d k \frac{1-\cos (k t)}{k^{2}+3 \alpha \widetilde{\sigma}(k)}, \quad \widetilde{\sigma}(k):=\frac{1}{3 \sqrt{2 \pi}} \int_{0}^{\infty} d t e^{-t} \frac{1-\cos (k t)}{F^{\frac{3}{2}}(t)} .
$$

Варианты этих интегральных уравнений были также получены, например, в работах $[15,20,21]$ для случая $d=3$. Результаты численных расчетов для $E_{0}(\alpha)$ и $m_{0}^{*}(\alpha)$ представлены в табл. 1 и 2.

Сделаем несколько замечаний.

1. Имеет место неравенство $J_{\beta}(\alpha, 0) \geqslant 1$. Следовательно, гауссово приближение дает ограничение сверху для точного значения ЭОС полярона с небольшой поправкой, слегка улучшаюшей фейнмановскую оценку: $E_{\mathrm{F}}(\alpha) \geqslant E_{0}(\alpha) \geqslant E(\alpha)$.

2 . Значение $E_{0}(\alpha)$ становится более точным в случае высших размерностей, поскольку $\lim _{d \rightarrow \infty} E_{0}(\alpha)=E(\alpha)$ вследствие асимптотического поведения

$$
\lim _{d \rightarrow \infty} J_{\beta}(\alpha, 0)=1 .
$$

3. Уравнение связи (10) допускает аналитическое решение в предельных случаях $\alpha \rightarrow$ 0 и $\alpha \rightarrow \infty$. При этом формулы (9) дают следующие асимптотические выражения:

$$
\begin{aligned}
& E_{0}(\alpha)= \begin{cases}-\alpha-\alpha^{2}\left(\frac{1}{4}-\frac{2}{3} \pi\right)-O\left(\alpha^{3}\right), & \alpha \rightarrow 0, \\
-\frac{\alpha^{2}}{\pi}-O(1), & \alpha \rightarrow \infty,\end{cases} \\
& m_{0}^{*}(\alpha)= \begin{cases}1+\frac{\alpha}{2}+\alpha^{2}\left(\frac{3}{2}-\frac{4}{\pi}\right)+O\left(\alpha^{3}\right), & \alpha \rightarrow 0, \\
\frac{16 \alpha^{4}}{\pi^{2}}+O\left(\alpha^{2}\right), & \alpha \rightarrow \infty .\end{cases}
\end{aligned}
$$


Фейнмановская вариационная модель воспроизводится, если мы ограничимся вместо точного решения $F(t)$ выбором двухпараметрической $(u, v)$ выпуклой комбинации асимптотических формул.

4. Для случая мгновенного взаимодействия мы решили эти уравнения численно.

\section{4. НЕГАУССОВЫ ПОПРАВКИ}

Для оценки негауссовых поправок к ЭОС и ЭМ полярона ограничимся рассмотрением полиномов второго порядка в разложении экспоненты в $J_{\beta}(\alpha, x)$. Учитывая только поправки второго порядка, для случаев слабого и сильного взаимодействий можно аналитически получить следующие выражения:

$$
\begin{aligned}
E_{0}(\alpha)+\Delta E_{2}(\alpha) & = \begin{cases}-\alpha-\alpha^{2}\left(\frac{3}{\sqrt{8}}-1\right)-O\left(\alpha^{3}\right), & \alpha \rightarrow 0, \\
-\alpha^{2} \cdot 0.327014-O(1), & \alpha \rightarrow \infty,\end{cases} \\
m_{0}^{*}(\alpha)+\Delta m_{2}^{*}(\alpha) & = \begin{cases}1+\frac{\alpha}{2}+\frac{\alpha^{2}(5 \sqrt{2}-4)}{16}+O\left(\alpha^{3}\right), & \alpha \rightarrow 0, \\
\alpha^{4} \cdot 1.858065+O\left(\alpha^{2}\right), & \alpha \rightarrow \infty .\end{cases}
\end{aligned}
$$

Для промежуточных значений константы взаимодействия $\alpha \sim 1$ мы решили эти уравнения численно. Мы получили, что негауссовы поправки второго порядка к ЭОС и ЭМ достигают своих максимумов (примерно 4 и $12 \%$ по сравнению с соответствующими гауссовыми членами) в точке $\alpha=1.93$. Таким образом, можно ожидать, что суммарное значение всех негауссовых поправок выше второго порядка не может сушественным образом изменить полученные для ЭОС и ЭМ полярона результаты, представленные в табл. 1 и 2.

\section{5. НИЗШИЕ СОСТОЯНИЯ ЛИБА-ЯМАДЗАКИ}

Нижние границы ЭОС и ЭМ полярона Фрёлиха $(d=3)$ были впервые определены Либом и Ямадзаки [22] в рамках гамильтонова формализма. Проводя аналогичную оценку для специального случая $d=1$, получаем

$$
\begin{aligned}
& E_{\mathrm{LY}}(\alpha)=\frac{1}{2}\left(-1+\sqrt{1-\frac{2 \alpha}{p}}-\alpha p\right), \\
& m_{\mathrm{LY}}^{*}(\alpha)=1+\frac{p^{2}-1}{p^{2}+1}\left(p^{4}+2 p^{2}-2\right),
\end{aligned}
$$

где $p$ - положительный корень уравнения $p^{3}(p-2 \alpha)-1=0$. Для случаев слабого и сильного взаимодействий уравнения (13) можно решить аналитически:

$$
\begin{aligned}
& E_{\mathrm{LY}}(\alpha)= \begin{cases}-\alpha-O\left(\alpha^{2}\right), & \alpha \rightarrow 0, \\
-\alpha^{2}+O(1), & \alpha \rightarrow \infty,\end{cases} \\
& m_{\mathrm{LY}}^{*}(\alpha)= \begin{cases}1+\frac{\alpha}{2}+O\left(\alpha^{2}\right), & \alpha \rightarrow 0, \\
16 \alpha^{4}+O\left(\alpha^{2}\right), & \alpha \rightarrow \infty .\end{cases}
\end{aligned}
$$

Результаты численных расчетов для $E_{\mathrm{LY}}(\alpha)$ и $m_{\mathrm{LY}}^{*}(\alpha)$ в области промежуточных значений константы связи, представленные в табл. 1 и 2 , можно сравнить с результатами, полученными в предыдущих разделах. 


\section{6. ЗАКЛЮЧЕНИЕ}

В эквивалентном гауссовому представлении (8) содержится стратегия получения нижней границы ЭОС дираковского полярона. Однако эта стратегия требует дополнительных усилий для строгого доказательства общего неравенства, и мы не будем обсуждать этот вопрос в настояшей статье. Вообше говоря, нахождение нижней границы ЭОС полярона требует методики, отличной от той, которой мы пользовались для оценок верхнейграницы. Мы хотели бы коснуться двух оценочных схем, которые позволяют сразу получать разумные (доказанные строго) значения нижних границ ЭОС полярона в размерности $d=1$.

Другой способ получить нижнюю границу ЭОС одномерного полярона методом ИТ заключается в том, чтобы следовать идее, предложенной в работе [23] для случая $d=3$. Можно заметить, что действие в уравнении (3) обладает запаздыванием (т.е. является нелокальным) и экспоненциально уменьшается при увеличении различия между двумя "временны́ми" переменными $t$ и $s$. Это делает модель неинтегрируемой, однако такое осложнение можно исправить ценой ухудшения точности. В частности, можно свести эту задачу к хорошо изученному локальному случаю. Таким образом, в случае дираковской поляронной модели необходимо выбирать евклидов пропагатор для близкодействуюшего потенциала типа $\delta$-функции [24], а не пропагатор, полученный для описываюшего атом водорода дальнодействующего взаимодействия кулоновского типа [23]. При этом нужно использовать набор нетривиальных функциональных неравенств, для того чтобы получить нижнюю границу ЭОС. Предварительная оценка показывает, что такой подход позволяет получить нижнюю гранищу, причем для случая слабого взаимодействия результат является разумным. К сожалению, оценки Либа-Ямадзаки для промежуточной и сильной связей будут, по-видимому, несколько хуже.

В заключение мы вычислили ЭОС и ЭМ дираковского полярона в размерностях $d>1$ в рамках и вне рамок (до членов второго порядка) обшего гауссова приближения, используя метод представлений, эквивалентных гауссовым. Мы показали, что ведущий гауссов вклад в энергию основного состояния немного уточняет фейнмановские оценки и принадлежит к самой нижней из возможных верхних границ. Негауссовы поправки следуюшего за ведушим порядка не нарушают значительно полученных результатов. Наш метод хорошо работает во всем диапазоне значений $\alpha$, он не требует значительного объема численных расчетов на суперкомпютерах [25], однако способен достаточно быстро дать надежные и состоятельные результаты. По сравнению с подходами, основанными на различных интерполяционных алгоритмах [26], наш подход также не имеет ни сингулярностей, ни сверхчувствительности, встречаюшихся в этих алгоритмах.

Благодарности. Я хотел бы поблагодарить Г. В. Ефимова, Х. Лешке и Р. Мюллера за ряд полезных обсуждений, а также фонд А. Гумбольдта за поддержку.

\section{Список литературы}

[1] L. D. Landau. Phys. Z. Sowjetunion. 1933. V. 3. P. 664.

[2] Н. Н. Боголюбов, С. В. Тябликов. ЖЭТФ. 1949. Т. 19. С. 256.

[3] H. Fröhlich. Philos. Mag. Suppl. 1954. V. 3. P. 325. 
[4] W. P. Su, J. R. Schrieffer, A. J. Heeger. Phys. Rev. Lett. 1980. V. 42. P. 1648; Phys. Rev. B. 1980. V. 22. P. 209.

[5] Z. B. Su, L. Yu. Phys. Rev. B. 1983. V. 27. P. 5199.

[6] K.-F. Berggren, T. J. Thornton, D. J. Newson, M. Pepper. Phys. Rev. Lett. 1986. V. 57. P. 1769.

[7] B. E. Sernelius. Phys. Rev. B. 1987. V. 36. P. 9509; 1988. V. 37. P. 7909

[8] F. M. Peeters, X. Wu, J. T. Devreese. Phys. Rev. B. 1986. V. 33. P. 3926.

[9] M. H. Degani, O. Hipólito, R. Lobo, G. A. Farias. J. Phys. C. 1986. V. 19. P. 2919.

[10] H. Leschke, S. Wonneberger. J. Phys. A. 1989. V. 22. P. L1009.

[11] C. Quinghu, W. Kelin, W. Shaolong. J. Phys.: Condens. Matter. 1994. V. 6. P. 6599.

[12] E. P. Gross. Ann. Phys. 1976. V. 99. P. 1.

[13] R. P. Feynman. Phys. Rev. 1955. V. 97. P. 660.

[14] F. M. Peeters, M. A. Smondyrev. Phys. Rev. B. 1991. V. 43. P. 4920.

[15] J. Adamowski, B. Gerlach, H. Leschke. Functional Integration. Theory and Application. New York: Plenum Press, 1980.

[16] G. V. Efimov, G. Ganbold. Phys. Part. Nucl. 1995. V. 26. P. 198.

[17] M. Dineykhan, G. V. Efimov, G. Ganbold, S. N. Nedelko. 1995 (unpublished).

[18] G. Ganbold, G. V. Efimov. Phys. Rev. B. 1994. V. 50. P. 373; Oscillator Representation in Quantum Physics (Lecture Notes in Physics, series Monographs m26). Berlin: Springer, 1995.

[19] G. Ganbold, G. V. Efimov. J. Phys.: Condens. Matter. 1998. V. 10. P. 4845.

[20] M. Saitoh. J. Phys. Soc. Japan. 1980. V. 49. P. 878.

[21] M. A. Smondyrev. Physica A. 1991. V. 171. P. 191.

[22] E. H. Lieb, K. Yamazaki. Phys. Rev. 1958. V. 111. P. 728.

[23] W. Fischer, H. Leschke, P. Müller. Path integration in quantum mechanics by changing drift and time of the underlying process. In: Path Integrals from meV to MeV. Proc. of the 4th Int. Conf. "Path Integrals from meV to MeV". Tutzing, May 18-21, 1992. Eds. H. Grabert, A. Inomata, L. S. Schulman, and U. Weiss. River Edge, New Jersey: World Scientific, 1993. P. 259-267.

[24] B. Gaveau, L. S. Schulman. J. Phys. A. 1986. V. 19. P. 1833.

[25] C. Alexandrou, R. Rosenfelder. Phys. Rep. 1992. V. 215. P. 1.

[26] H. Kleinert. Phys. Lett. A. 1995. V. 207. P. 133. 